\title{
Modified high-flow nasal cannula for children with respiratory distress
}

\author{
Sarocha Itdhiamornkulchai, MD ${ }^{1}$, Aroonwan Preutthipan, MD, FCCP ${ }^{2}$, Jarin Vaewpanich, MD ${ }^{1}$, Nattachai Anantasit, MD ${ }^{1}$ \\ ${ }^{1}$ Division of Pediatric Critical Care Medicine, Department of Pediatrics, Faculty of Medicine, Ramathibodi Hospital, Mahidol University, Bangkok, Thailand; ${ }^{2}$ Division of \\ Pediatric Pulmonology, Department of Pediatrics, Faculty of Medicine, Ramathibodi Hospital, Mahidol University, Bangkok, Thailand
}

Background: High-flow nasal cannula (HFNC) is a noninvasive respiratory support that provides the optimum flow of an air-oxygen mixture. Several studies demonstrated its usefulness and good safety profile for treating pediatric respiratory distress patients. However, the cost of the commercial HFNC is high; therefore, the modified high-flow nasal cannula was developed. Purpose: This study aimed to compare the effectiveness, safety, and nurses' satisfaction of the modified system versus the standard commercial HFNC.

Methods: This prospective comparative study was performed in a tertiary care hospital. We recruited children aged 1 month to 5 years who developed acute respiratory distress and were admitted to the pediatric intensive care unit. Patients were assigned to 2 groups (modified vs. commercial). The effectiveness and safety assessments included vital signs, respiratory scores, intubation rate, adverse events, and nurses' satisfaction.

Results: A total of 74 patients were treated with HFNC. Thirtynine patients were assigned to the modified group, while the remaining 35 patients were in the commercial group. Intubation rate and adverse events did not differ significantly between the 2 groups. However, the commercial group had higher nurses' satisfaction scores than the modified group.

Conclusion: Our findings suggest that our low-cost modified HFNC could be a useful respiratory support option for younger children with acute respiratory distress, especially in hospital settings with financial constraints.

Key words: High-flow nasal cannula, Noninvasive respiratory support, Respiratory distress, Respiratory failure

\section{Key message}

Question: Can the modified high-flow nasal cannula (HFNC) provide alternative respiratory support for children with acute respiratory distress?

Finding: A total of 74 patients were assigned to the modified or commercial HFNC groups. The intubation rate, length of hospital stay, and adverse events did not differ between the 2 groups.
Meaning: The modified HFNC can provide alternative respiratory support for pediatric respiratory distress.

\section{Introduction}

High-flow nasal cannula (HFNC) is noninvasive respiratory support that provides optimum flow of heated, humidified, and air-oxygen mixture to patients via a nasal cannula interface. The traditional nasal cannula uses a flow rate of fewer than $4 \mathrm{~L}$ per minute $(\mathrm{L} / \mathrm{min})$ and is associated with drying of the airway mucosa and discomfort. ${ }^{1)}$ The optimal flow rate is unknown, but it is adjusted individually to minimize the patients' work of breathing. High flow is usually defined as flow rate varied from $2-8 \mathrm{~L} / \mathrm{min}$ in infants and range up to $60 \mathrm{~L} / \mathrm{min}$ in adolescents and adults. It supplies adequate warmed and humidified gas that decreases pulmonary conductance. ${ }^{2-4)}$ The mechanisms of HFNC include washout of nasopharyngeal dead space, facilitated secretion removal, decreased inspiratory resistance, increased pulmonary compliance, and decreased work of breathing. ${ }^{5,6)}$ Many studies indicated HFNC is useful in pediatric respiratory distress patients with good safety profiles. $\left.{ }^{6-9}\right)$ A recent study with large randomized controlled trial of HFNC in infants with bronchiolitis showed HFNC had significantly lower rate of treatment failure when compared with standard oxygen therapy. ${ }^{10)}$ However, some studies reported mild to severe adverse effects which included epistaxis, abdominal distention, and air leak syndrome. ${ }^{7,11)}$ The commercial HFNC (AIRVO II, Fisher \& Paykel Healthcare, Auckland, New Zealand) is used worldwide in hospitals. However, its cost is relatively high for routine use in developing countries and most patients cannot afford this device. Therefore, our center has developed a modified HFNC since 2011 that costs of approximately one-third of the commercial HFNC. Our recent retrospective study showed the modified HFNC was useful respiratory support in young children with community-acquired pneumonia. ${ }^{12)}$ The aim of this study was to compare the effectiveness and safety of the commercial and modified HFNC in children with respiratory distress.

Corresponding author: Nattachai Anantasit, MD, Division of Pediatric Critical Care, Department of Pediatrics, Ramathibodi Hospital, 270 Rama VI Road, Ratchathewi, Bangkok 10400, Thailand

凶Email: nattachai032@hotmail.com, https://orcid.org/0000-0002-2770-1718

Received: 14 August, 2020, Revised: 24 April, 2021, Accepted: 13 May, 2021

This is an open-access article distributed under the terms of the Creative Commons Attribution Non-Commercial License (http://creativecommons.org/licenses/bync/4.0/) which permits unrestricted non-commercial use, distribution, and reproduction in any medium, provided the original work is properly cited.

Copyright (c) 2022 by The Korean Pediatric Society 

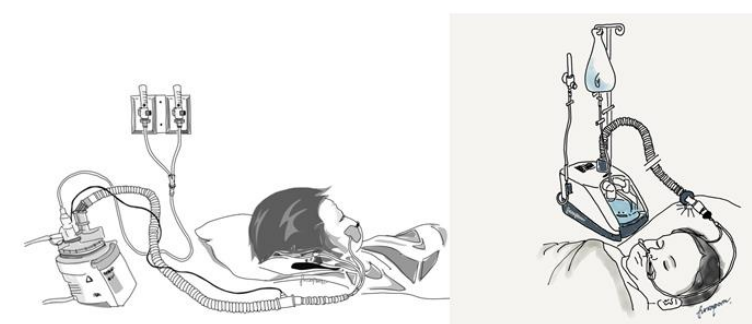

\begin{tabular}{|l|c|c|c|}
\hline & Modified HFNC ( $\mathbf{n = 3 9 )}$ & Commercial HFNC (n=35) & P value \\
\hline Intubation rate, $n(\%)$ & $4(10.2)$ & $7(20.0)$ & 0.330 \\
\hline Length of hospital stay, days & $15(8-39)$ & $17(9-42)$ & 0.645 \\
\hline $\begin{array}{l}\text { Adverse events, } \mathrm{n}(\%) \\
\quad \text { Nasal redness }\end{array}$ & $1(2.6)$ & $1(2.8)$ & 0.386 \\
\hline Epitaxis & $1(2.6)$ & $1(2.8)$ & \\
Bloating & $3(7.7)$ & $6(17.1)$ & \\
\hline Vomiting & $1(2.6)$ & $1(2.8)$ & \\
\hline
\end{tabular}

Graphical abstract. Comparison of the modified and commercial high-flow nasal cannula (HFNC) systems.

\section{Methods}

We performed a single-center, prospective comparative study conducted in total 17-bed multidisciplinary medical-surgical pediatric intensive care unit and intermediate ward in our hospital from February 2017 to February 2018. Our institute is a regional referral center for pediatric subspecialty care in Thailand. This study was approved by the Human Research Ethics Committee of Ramathibodi Hospital, Mahidol University Faculty of Medicine (IRB number; MURA2016/575), and informed consent was obtained. Eligible subjects included all patients aged 1 month to 5 years old who developed respiratory distress and required HFNC. Patients who had facial anomaly, tracheostomy, respiratory failure, or do-not-resuscitate status were excluded from this study. Participants were assigned to either the modified group (using modified HFNC) and the commercial group (using AIRVO II). We collected demographic data, vital signs, the modified respiratory score, complications, length of hospital stay, and intubation in both groups.

\section{Study protocol}

Eligible patients with parental consent were included. All patients' nostrils were cleaned by normal saline and nasal decongestant before the application of HFNC. Due to the inadequate availability of the commercial HFNC during the initial phase for 2 months (from February to April 2017), the patients who were admitted during this period were allocated to the modified HFNC group. Once the commercial HFNC was readily available, the allocation was made using 1:1 alternate allocation into each group based on the admitting diagnosis. The analysis was performed strictly per protocol and no cross-over was allowed. The nasal cannula size was used as per the current manufacturer's instructions in the commercial group, while the modified group used nasal cannula size approximately $50 \%-70 \%$ fit to the patient's

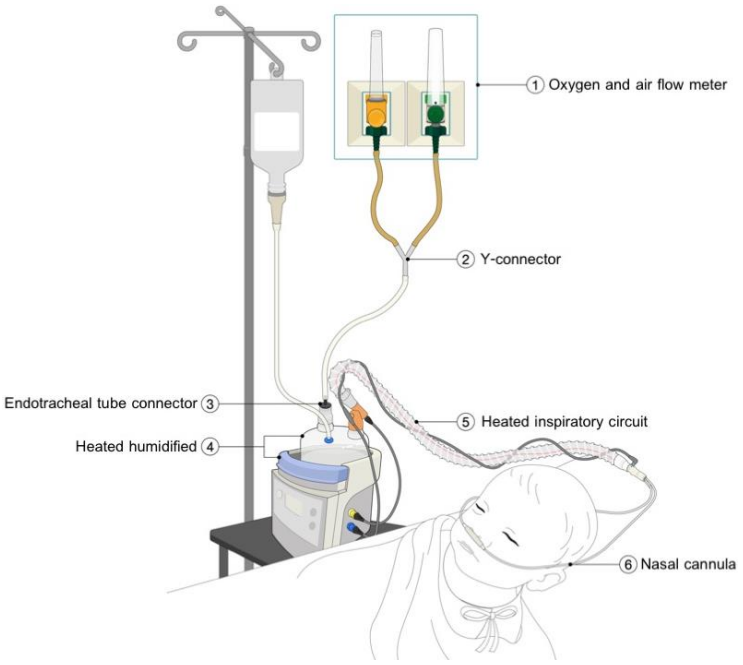

Fig. 1. Drawing of the modified high-flow nasal cannula system. (1) Oxygen and air flow meter. (2) Y-connector. (3) Endotracheal tube connector. (4) Heated humidified. (5) Heated inspiratory circuit. (6) Nasal cannula.

nostrils for allowing leakage of excessive flow. The humidifier was auto set to $37^{\circ} \mathrm{C}$. The modified HFNC device includes oxygen and air pipelines, a heated humidifier (MR850, Fisher \& Paykel Healthcare), a single-heated breathing circuit, and standard oxygen nasal cannula as shown in Fig. 1. The modified respiratory clinical score (MRCS) was used to assess the severity of respiratory distress. The components included 4 parameters (respiratory rate scale, $1-3$; retractions, dyspnea, and wheezing scale, $0-3)$. The maximum for this score was $12 \cdot{ }^{13,14)}$ The initial flow rate of HFNC was adjusted by patient's age: 1-6 months, 4-8 L/min; 6-18 months, 4-12 L/min; 18-60 months, 8-15 L/ min; and maximal initial flow rate did not exceed $2 \mathrm{~L} / \mathrm{kg} / \mathrm{min}$ with $\mathrm{FiO}_{2} 40 \%{ }^{15}$ ) Flow rate was increased to $2-3 \mathrm{~L} / \mathrm{kg} / \mathrm{min}$ or maximum $20 \mathrm{~L} / \mathrm{min}$ and/or increased $\mathrm{FiO}_{2}$ to $50 \%$ after 15 minutes if there was no clinical improvement or the MRCS was 
higher than 3 . We measured the vital signs, oxygen saturation, and the MRCS at initial, 1, 4, 12, 24, and 48 hours. Other respiratory care and nursing care were similar in both groups. When the patient was clinically stable, $\mathrm{FiO}_{2}$ was weaned to $40 \%$ then flow rate of HFNC was reduced $2-4 \mathrm{~L} /$ min every 2 hours until flow rate of $4-6 \mathrm{~L} / \mathrm{min}$ was achieved.

Nurses' satisfaction in using these 2 systems' devices were assessed and filled-in in Likert scale (from " $1=$ completely disagree" to " $5=$ in total agreement") with 6 topics including endurance of equipment, easy to assemble, easy to use, patient comfort, noise, and easy to clean.

\section{Definitions}

Indications for using HFNC were children who had respiratory distress and failed to respond to low flow nasal cannula.

The intubation criteria were defined as persistent respiratory acidosis (arterial $\mathrm{pH}<7.35$ with $\mathrm{PaCO}_{2}>45 \mathrm{mmHg}$ ) after using HFNC for at least 2 hours, persistent hypoxemia $\left(\mathrm{SpO}_{2}\right.$ $<90$ or $\mathrm{PaO}_{2}<60$ mmHg during treatment with $\mathrm{FiO}_{2}>50 \%$ ), or had clinical signs of acute respiratory failure (respiratory rate $>2$ standard deviation for age, increased respiratory effort such as accessory muscle use, intercostal retraction, or paradoxical motion of the abdomen). ${ }^{16)}$

The definition of respiratory distress was defined as patients who were receiving low flow nasal cannula and had signs of respiratory distress, including an increase in respiratory rate, dyspnea, grunting, nasal flaring, or chest retraction.

\section{Study outcomes}

The primary outcome was the proportion of patients who required endotracheal tube intubation. The secondary outcomes were the length of hospital stay, vital signs, the MRCS, and complications related to HFNC.

\section{Statistical analysis}

Statistical analysis was performed using IBM SPSS Statistics ver. 20.0 (IBM Co., Armonk, NY, USA). Data were presented as the mean and standard deviation, median and interquartile range were reported. The comparison of data between the 2 groups used chi-square or Fisher exact test for categorical variables. The Student $t$ test was used for continuous data with normal distribu-

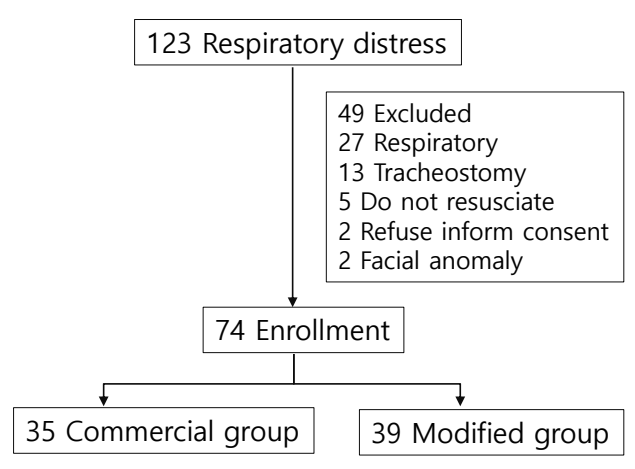

Fig. 2. Flow diagram of the study patient enrollment process. tion or Mann-Whitney $U$ test for continuous data without normal distribution. A $P$ value of less than 0.05 was considered statistically significant.

\section{Results}

Altogether 123 respiratory distress patients were screened. Forty-nine patients were excluded as in Fig. 2. A total of 74 children met the inclusion criteria and were commenced on HFNC therapy. The median age was 16 months (interquartile range [IQR], 6-26), and 35 males (47.3\%) were enrolled. Thirty-nine patients were assigned into the modified group, while the remaining 35 patients were in the commercial group. The baseline characteristics were not significantly different between the 2 groups (Table 1).

There were 12 patients (16.2\%) who needed the escalating respiratory support after using HFNC which included 1 patient who used noninvasive positive pressure ventilation and the remaining patients required intubation. The overall intubation rate was $14.8 \%$. Four out of 39 patients $(10.2 \%)$ in the modified group and 7 out of 35 patients (20.0\%) in the commercial group $(P=0.330)$ required intubation which occurred within 48 hours after use of HFNC. All patients discharged home safely. The median lengths of hospital stay were 15 (IQR, 8-39) and 17 days (IQR, 9-42) in the modified and commercial groups, respectively $(P=0.645)$ as shown in Table 2. Patients in both groups showed improvement in heart rate, respiratory rate, and the MRCS after

Table 1. Baseline characteristics of the study patients

\begin{tabular}{|c|c|c|c|}
\hline Characteristic & $\begin{array}{l}\text { Modified group } \\
\quad(n=39)\end{array}$ & $\begin{array}{l}\text { Commercial } \\
\text { group }(n=35)\end{array}$ & $P$ value \\
\hline Age (mo) & $16(4.5-29)$ & $15(7-26)$ & 0.710 \\
\hline Male sex & $17(43.6)$ & $18(51.4)$ & 0.500 \\
\hline Body weight $(\mathrm{kg})$ & $8.7(5.2-12.8)$ & $9.2(7.0-10.8)$ & 0.866 \\
\hline Height (cm) & $75(60-86)$ & $76(69-84)$ & 0.618 \\
\hline Positive viral infection & $17(43.6)$ & $16(45.7)$ & 0.358 \\
\hline Pre-existing comorbidity & & & 0.368 \\
\hline None & $9(23.1)$ & $15(42.8)$ & \\
\hline Congenitial heart diseases & $8(20.5)$ & $3(8.6)$ & \\
\hline Chronic lung diseases & $9(23.1)$ & $7(20.0)$ & \\
\hline Others & $13(33.3)$ & $10(28.6)$ & \\
\hline Diagnosis & & & 0.868 \\
\hline Pneumonia & $15(38.4)$ & $11(31.4)$ & \\
\hline Postextubation stridor & $14(35.9)$ & $16(45.7)$ & \\
\hline Bronchiolitis & $4(10.3)$ & $3(8.6)$ & \\
\hline Pulmonary edema & $2(5.1)$ & $2(5.7)$ & \\
\hline Atelectasis & $1(2.6)$ & $1(2.9)$ & \\
\hline Others & $3(7.7)$ & $2(5.7)$ & \\
\hline Initial heart rate (bpm) & $145(128-168)$ & $145(120-168)$ & 0.595 \\
\hline Initial respiratory rate & $44(36-48)$ & $36(30-48)$ & 0.076 \\
\hline Initial MRCS & $5(2-6)$ & $4(2-6)$ & 0.398 \\
\hline Initial SpO2 & $100(97-100)$ & $100(99-100)$ & 0.234 \\
\hline
\end{tabular}



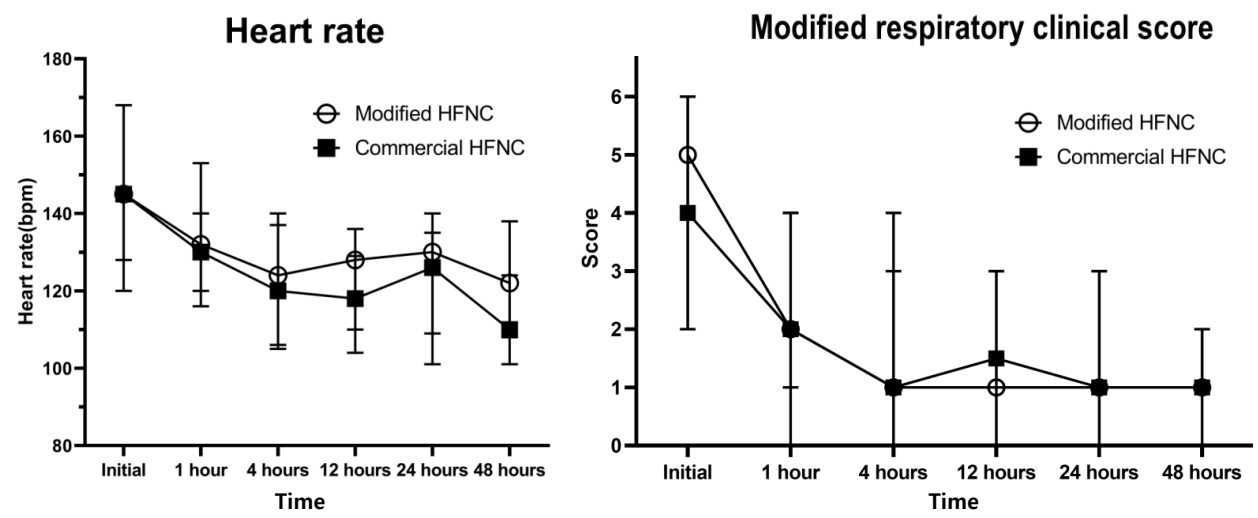

Fig. 3. Heart rate and modified respiratory clinical scores by group. HFNC, high-flow nasal cannula.

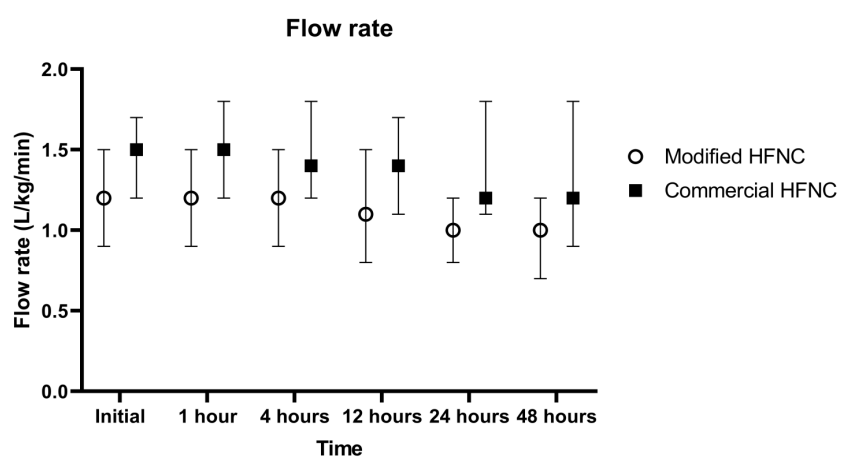

Fig. 4. Flow rate by group. HFNC, high-flow nasal cannula.

enrollment (Fig. 3), and the changes of heart rate, respiratory rate, and the MRCS were not significantly different between the 2 groups. The flow rate of HFNC per patient's weight in the modified group was statistically significantly lower than the commercial group at initial, 4 hours, 12 hours, 24, and 48 hours as shown in Fig. 4. There were some minor adverse events in both groups such as nasal redness, epistaxis, bloating, vomiting, but no statistical difference between the groups $(P=0.386)$ as shown in Table 2. No serious adverse events occurred in this study. The nurses' satisfaction was collected from 69 nurses during this study. The commercial HFNC had significantly more satisfaction than the modified HFNC in terms of easy to assemble, easy to use, patient comfort, and noise $(P<0.05)$ as shown in Table 2 . As the heterogenesity of cause of intubation, we had post hoc analysis without patients who had postextubation stridor. There were 44 patients, 25 patients in the modified group, and 19 patients in the commercial group. The baseline characteristic were not different between the 2 groups. Three out of 25 patients (12\%) in the modified group and 3 out of 19 patients (15.8\%) in the commercial group $(P=0.717)$ required intubation which occurred within 48 hours after use of HFNC. The length of hospital stay, duration of HFNC, and adverse events were not different between the 2 groups.
Table 2. Clinical outcomes, complications, and nurses' satisfaction scores of modified versus commercial HFNC systems

\begin{tabular}{lccr}
\hline Variable & $\begin{array}{c}\text { Modified group } \\
(\mathrm{n}=39)\end{array}$ & $\begin{array}{c}\text { Commercial } \\
\text { group }(\mathrm{n}=35)\end{array}$ & $P$ value \\
\hline Variable & $4(10.2)$ & $7(20.0)$ & 0.330 \\
Intubation & $15(8-39)$ & $17(9-42)$ & 0.645 \\
Length of hospital stay (day) & & & 0.386 \\
Adverse events & & & \\
Nasal redness & $1(2.6)$ & $1(2.8)$ & \\
Epitaxis & $1(2.6)$ & $1(2.8)$ & \\
Bloating & $3(7.7)$ & $6(17.1)$ & \\
Vomiting & $1(2.6)$ & $1(2.8)$ & \\
Duration of HFNC (hr) & $75(32-115)$ & $62(29-139)$ & 0.916 \\
Nurses' satisfaction & & & \\
Endurance of equipment & $3.96 \pm 0.55$ & $3.88 \pm 0.63$ & 0.520 \\
Easy to assemble & $3.83 \pm 0.64$ & $4.25 \pm 0.58$ & $<0.001$ \\
Easy to use & $4.03 \pm 0.57$ & $4.28 \pm 0.57$ & 0.013 \\
Easy to clean & $4.01 \pm 0.58$ & $4.09 \pm 0.53$ & 0.516 \\
Noise & $3.46 \pm 0.72$ & $4.26 \pm 0.67$ & $<0.001$ \\
Patient comfort & $3.81 \pm 0.69$ & $4.16 \pm 0.63$ & 0.003 \\
\hline
\end{tabular}

Values are number (\%), median (interquartile range), or mean \pm standard deviation.

HFNC, high-flow nasal cannula.

Boldface indicates a statistically significant difference with $P<0.05$ by Mann-Whitney U test.

\section{Discussion}

In this study, we investigated the effectiveness and safety of our modified HFNC compared to the commercial HFNC in pediatric patients with respiratory distress. Our data suggest that modified HFNC might be effective and safe to be used in hospitalized pediatric patients as an alternative respiratory support. To our knowledge, this study is the first comparative study of the modified HFNC with the commercial HFNC for pediatric respiratory distress in resource-limited hospital settings. We had the experimental study prior this study by using the nasal model and measured the upper airway pressure with the different flow from $3 \mathrm{~L} / \mathrm{min}$ then upto $25 \mathrm{~L} / \mathrm{min}$ on the modified HFNC and com. mercial HFNC. The upper airway pressure was between 1.4-7.5 $\mathrm{cmH}_{2} \mathrm{O}$ on both machines. Before this study, we had measured 
the pharyngeal airway pressure on the modified HFNC in 3 young bronchiolitis children with mouth closure. The pharyngeal pressure with flow $1 \mathrm{~L} / \mathrm{kg} / \mathrm{min}$ and $1.5 \mathrm{~L} / \mathrm{kg} / \mathrm{min}$ were $1-2.7$ and $1.4-4 \mathrm{cmH}_{2} \mathrm{O}$, respectively. This result was similar with the previous study. ${ }^{17)}$

Previous studies showed the intubation rate in patients with acute bronchiolitis, respiratory distress, or postextubation stridor patients varied from $7 \%-40 \% .{ }^{18-21)}$ The intubation rate in bronchiolitis patients with HFNC therapy was lower than others diseases. ${ }^{10,18,19)}$ The intubation rate in this study was $14.8 \%$. The 2 most common conditions requiring HFNC in this study were pneumonia and postextubation stridor (56 of 74 patients, $75.7 \%)$. Although the intubation rate of the modified group was approximately half of the commercial group, the difference did not reach statistical significance, probably due to small sample size. Other clinical outcomes were not different between the 2 groups. Both the modified and commercial HFNC had comparable beneficial effect on improving heart rate, and MRCS. The adverse events were not different between the 2 groups.

The nurses preferred to use the commercial HFNC because it was easy to assemble, and easy to use. However, the cost of modified HFNC is much lower than the commercial HFNC. The costs for the commercial HFNC machine and its disposable set is 7,100 and 190 United States dollar (USD), respectively, while those of the modified HFNC is 2,500 and 25 USD (calculated by $1 \mathrm{USD}=32$ Thai baht). The limitations of the modified HFNC are this system requires the oxygen and air pipeline for the source of flow, and it can be used only in hospital setting. In addition, the maximal flow rate of the modified HFNC is limited up to 20 $\mathrm{L} / \mathrm{min}$ because the connection system burst and broke when the flow rate was higher than $20 \mathrm{~L} / \mathrm{min}$. Therefore, it cannot be used in older children who need higher flow rate.

This study has some limitations. This was a prospective com. parative single-center study with a relatively small sample size. We planed to do the randomized controlled trial study at the beginning of the study, but it was not practical due to limited availability of the commercial HFNC machine during the study period. This might cause selection bias. We minimized this potential bias by using the stratification by patients' diagnosis and strictly followed the study protocol in both groups. In the further study, the randomized controlled trial will be conducted.

In conclusion, we suggest that the modified HFNC could be an alternative respiratory support for children with acute respiratory distress in limited-resourced hospital setting.

\section{Footnotes}

Funding: The author(s) received financial support for the research from Ramathibodi Research Fund.

Conflicts of interest: No potential conflict of interest relevant to this article was reported.
Acknowledgments: The authors would like to acknowledge all colleagues in Pediatric Critical Care and Pulmonary Division, the Department of Pediatrics Ramathibodi Hospital, Mahidol University. We would also like to thank Prof. Pat Mahachoklertwattana for the critical review of the article and Dr. Jeeraparn Phosuwattanakul for drawing the modified high-flow nasal cannula picture. We would like to thank Mr. Stephen Pinder for grammar correction. Furthermore, our special thanks to all patients who participated in this study.

ORCID:

Nattachai Anantasit 1 https://orcid.org/0000-0002-2770-1718

\section{References}

1. Ward JJ. High-flow oxygen administration by nasal cannula for adult and perinatal patients. Respir Care 2013;58:98-122.

2. Dysart K, Miller TL, Wolfson MR, Shaffer TH. Research in high flow the rapy: mechanisms of action. Respir Med 2009;103:1400-5.

3. Bressan S, Balzani M, Krauss B, Pettenazzo A, Zanconato S, Baraldi E. High-flow nasal cannula oxygen for bronchiolitis in a pediatric ward: a pilot study. Eur J Pediatr 2013;172:1649-56.

4. Bueno Campana M, Olivares Ortiz J, Notario Munoz C, Rupérez Lucas M, Fernández Rincón A, Patiño Hernández O, et al. High flow therapy versus hypertonic saline in bronchiolitis: randomised controlled trial. Arch Dis Child 2014;99:511-5.

5. Mikalsen IB, Davis P, Oymar K. High flow nasal cannula in children: a literature review. Scand J Trauma Resusc Emerg Med 2016;24:93.

6. Kwon JW. High-flow nasal cannula oxygen therapy in children: a clinical review. Clin Exp Pediatr 2020;63:3-7.

7. Ten Brink F, Duke T, Evans J. High-flow nasal prong oxygen therapy or nasopharyngeal continuous positive airway pressure for children with moderate-to-severe respiratory distress?*. Pediatr Crit Care Med 2013; 14:e326-31.

8. Hutchings FA, Hilliard TN, Davis PJ. Heated humidified high-flow nasal cannula therapy in children. Arch Dis Child 2015;100:571-5.

9. Kawaguchi A, Yasui Y, deCaen A, Garros D. The clinical impact of heated humidified high-flow nasal cannula on pediatric respiratory distress. Pediatr Crit Care Med 2017;18:112-29.

10. Franklin D, Babl FE, Schlapbach LJ, Oakley E, Craig S, Neutze J, et al. A randomized trial of high-flow oxygen therapy in infants with bronchiolitis. NEngl J Med 2018;378:1121-31.

11. Hegde S, Prodhan P. Serious air leak syndrome complicating high-flow nasal cannula therapy: a report of 3 cases. Pediatrics 2013;131:e939-44.

12. Vareesunthorn I, Preutthipan A. Modified high-flow nasal cannula in young children with pneumonia: a 3-year retrospective study. Pediatr Respirol Crit Care Med 2018;2:45-50.

13. Kaya Z, Turktas I. Correlation of clinical score to pulmonary function and oxygen saturation in children with asthma attack. Allergol Immunopathol 2007;35:169-73.

14. Liu LL, Gallaher MM, Davis RL, Rutter CM, Lewis TC, Marcuse EK. Use of a respiratory clinical score among different providers. Pediatr Pulmonol 2004;37:243-8.

15. Riese J, Fierce J, Riese A, Alverson BK. Effect of a hospital-wide high-flow nasal cannula protocol on clinical outcomes and resource utilization of bronchiolitis patients admitted to the PICU. Hosp Pediatr 2015;5:613-8.

16. Malhotra D, Gurcoo S, Qazi S, Gupta S. Randomized comparative efficacy of dexamethasone to prevent postextubation upper airway complications in children and adults in ICU. Indian J Anaesth 2009;53:442-9.

17. Arora B, Mahajan P, Zidan MA, Sethuraman U. Nasopharyngeal airway pressures in bronchiolitis patients treated with high-flow nasal cannula oxygen therapy. Pediatr Emer Care 2012;28:1179-84. 
18. Schibler A, Pham TM, Dunster KR, Foster K, Barlow A, Gibbons K, et al. Reduced intubation rates for infants after introduction of high-flow nasal prong oxygen delivery. Intensive Care Med 2011;37:847-52.

19. McKiernan C, Chua LC, Visintainer PF, Allen H. High flow nasal cannulae therapy in infants with bronchiolitis. J Pediatr 2010;156:634-8.

20. Wraight TI, Ganu SS. High-flow nasal cannula use in a paediatric intensive care unit over 3 years. Crit Care Resusc 2015;17:197-201.

21. Long E, Babl FE, Duke T. Is there a role for humidified heated high-flow nasal cannula therapy in paediatric emergency departments? Emerg Med J2016;33:386-9.
How to cite this article: Itdhiamornkulchai $S$, Preutthipan A, Vaewpanich J, Anantasit N. Modified high-flow nasal cannula for children with respiratory distress. Clin Exp Pediatr 2022;65:136-41. https://doi.org/10.3345/cep.2020.01403 